\title{
A Spontaneous Segmental Deletion from Chromosome Arm 3DL Enhances Fusarium Head Blight Resistance in Wheat
}

\begin{tabular}{|r|l|}
\hline Journal: & Genome \\
\hline Manuscript ID & gen-2015-0088 \\
\hline Manuscript Type: & Article \\
\hline Date Submitted by the Author: & 14-Jul-2015 \\
\hline Complete List of Authors: & $\begin{array}{l}\text { Garvin, David; USDA-ARS, Plant Science Research Unit, University of } \\
\text { Minnesota } \\
\text { Porter, Hedera; University of Minnesota, Plant Pathology } \\
\text { Blankenheim, Zachary; USDA-ARS, Plant Science Research Unit } \\
\text { Chao, Shiaoman; USDA-ARS Biosciences Research Lab, } \\
\text { Dill-Macky, Ruth; University of Minnesota, Plant Pathology }\end{array}$ \\
\hline Keyword: & Fusarium, Susceptibility, Breeding, Genetics, Mapping \\
\hline & \\
\hline
\end{tabular}

SCHOLARONE ${ }^{\text {It }}$

Manuscripts 


\section{A Spontaneous Segmental Deletion from Chromosome Arm 3DL Enhances Fusarium Head Blight Resistance in Wheat}

David F. Garvin $^{1 *}$, Hedera Porter ${ }^{2}$, Zachary J. Blankenheim ${ }^{1}$, Shiaoman Chao ${ }^{3}$, and Ruth Dill$\mathrm{Macky}^{2}$

${ }^{1}$ USDA-ARS Plant Science Research Unit, St. Paul, MN 55108; ${ }^{2}$ Dept. of Plant Pathology, University of Minnesota, St. Paul, MN 55108; ${ }^{3}$ USDA-ARS Biosciences Research Laboratory, Fargo, ND. *Corresponding author (David.Garvin@ars.usda.gov). 


\begin{abstract}
Much effort has been directed at identifying sources of resistance to Fusarium head blight (FHB) in wheat. We sought to identify molecular markers for what we hypothesized was a new major FHB resistance locus originating from the wheat cultivar 'Freedom' and introgressed into the susceptible wheat cultivar 'USU-Apogee'. An $F_{2: 3}$ mapping population from a cross between Apogee and $\mathrm{A} 30$, its $\mathrm{BC}_{4}$ - near-isoline exhibiting improved $\mathrm{FHB}$ resistance, was evaluated for resistance. The distribution of FHB resistance in the population approximated a 1:3 moderately resistant:moderately susceptible+susceptible ratio. Separate disease evaluations established that A30 accumulated less deoxynivalenol and yielded a greater proportion of sound grain than Apogee. Molecular mapping revealed that the FHB resistance of A30 is associated with molecular markers on chromosome arm 3DL that exhibit a null phenotype in A30 but are present in both Apogee and Freedom, indicating a spontaneous deletion occurred during the development of A30. Aneuploid analysis revealed that the size of the deleted segment is approximately $19 \%$ of the arm's length. Our results suggest that the deleted interval of chromosome arm 3DL in Apogee may harbor FHB susceptibility genes that promote disease spread in infected spikes, and that their elimination increases FHB resistance in a novel manner.
\end{abstract}

Key words: Fusarium, susceptibility, breeding, genetics, mapping 


\section{Introduction}

The disease Fusarium head blight (FHB), caused by members of the fungal genus Fusarium, reduces yields and erodes grain quality in wheat and other small grains. The principal species causing FHB in the U.S. is F. graminearum Schwabe (Stack and McMullen 1985). While FHB has been recognized as a disease of wheat in the U.S. for over a century, severe epidemics have impacted all wheat classes and most wheat production regions in the U.S. since the early 1990s (McMullen et al. 1997; Windels 2000). Today FHB is the most damaging disease facing the wheat industry. Historically, the upper Midwest hard red spring wheat production region has incurred the largest losses to FHB, but the geographic area in which the disease is affecting wheat production is growing significantly (McMullen et al. 2012), with Idaho being the most recent state to confront the challenges posed by FHB.

Progress in developing FHB-resistant wheat varieties is challenging due to the fact that the genetics of resistance is complex (Bai and Shaner 2004). Under field conditions, a diverse range of environmental factors and their interactions with plant genotypes can cause problems with accurately phenotyping reactions (Groth et al. 1999; Miedaner et al. 2001). Greenhouse testing for FHB resistance largely has been mostly useful for evaluating resistance to spread of the disease in the spike (Type II resistance) (Schroeder and Christensen 1963).

To assist FHB resistance breeding efforts, a proliferation of FHB resistance quantitative trait locus (QTL) mapping research in wheat around the world has led to the identification of more than 200 QTLs distributed on all 21 wheat chromosomes (Buerstmayr et al. 2009, Liu et al. 2009; Loffler et al. 2009). However, despite this intensive international effort, only Fhb1, the most broadly validated FHB resistance QTL (Buerstmayr et al. 2009), is widely employed by 
U.S. and international wheat breeding programs. A small number of additional wheat QTLs have recently been "Mendelized" into single locus traits in a fashion similar to Fhbl (Cuthbert et al. 2007; Xue et al. 2010), and they may hold promise for breeding programs as well. A host of other QTLs have not been validated, or only partially so (Buerstmayr et al. 2009; Liu et al. 2009; Loffler et al. 2009), and together with their generally small effects, are thus unlikely to be broadly utilized in breeding programs.

After many years of genetics analysis and breeding research, it is evident that the effect of a FHB resistance QTL can vary significantly depending on the genetic background in which it is present. Pumphrey et al. (2007) and Salameh et al. (2011) both reported that genetic background affected the level of resistance associated with Fhb1. Similarly, introgression of Fhbl into durum wheat does not enhance resistance (H. Buerstmayr, personal communication, 2013), nor does it improve resistance in triticale (D.F. Garvin, unpublished). Modifiers of FHB resistance, including susceptibility genes and resistance suppressor genes, are postulated to contribute to the observation that genetic background affects the expression of FHB resistance QTL, but scant effort has been devoted to exploring the genetic basis of these phenomena. Basnet et al. (2012) proposed that Sumai 3 has a susceptibility gene on chromosome 2DS, while Srinivasachary et al. (2008) found that compromised FHB resistance was associated with the dwarfing gene $R h t-D 1 b$ but was not due to height per se. In tetraploid wheat, the first FHB resistance QTL to be described (Otto et al. 2002) surprisingly derives from a highly susceptible genotype (Stack et al. 2002). Lastly, Ma et al. (2006) reported that the loss of certain wheat chromosome arms increased FHB resistance. But beyond this, little is known about the genetics of FHB resistance suppression and FHB susceptibility even though they may be significant determinants of QTL 
penetrance and expressivity, and may lead to the development of complementary strategies for FHB resistance improvement by eliminating genes that are involved in FHB susceptibility. In this study we report the genetic characterization of improved FHB resistance in a nearisogenic line of the highly susceptible wheat cv Apogee (Mackintosh et al. 2006), and through molecular mapping demonstrate that the resistance is not due to the presence of a postulated new FHB resistance QTL, but rather to a spontaneous deletion of a chromosome segment of chromosome arm 3DL that may eliminate a gene or genes involved in FHB susceptibility.

\section{Materials and methods}

Plant materials: The rapid-maturing dwarf hard red spring wheat cv 'USU-Apogee' (Bugbee et al. 1997) (Apogee) and an Apogee near-isoline (NIL) were employed in this study. Previous research has revealed that Apogee is highly susceptible to FHB (Mackintosh et al. 2006). 'A30' is a $\mathrm{BC}_{4} \mathrm{~F}_{2}$-derived line of Apogee developed during an effort to introgress a putative FHB resistance QTL in the soft red winter wheat cultivar 'Freedom' postulated to reside on chromosome 2A (Gupta 2002). A30 is homozygous for the Freedom allele of SSR marker Xgwm296 that was reported to be associated with this FHB resistance QTL (Gupta 2002). Greenhouse disease evaluations of A30 revealed a consistent pattern of improved FHB resistance compared to Apogee, while several other independently derived $\mathrm{BC}_{4} \mathrm{~F}_{2}$-derived near-isolines of Apogee with the Freedom allele of Xgwm296 did not display improved resistance (data not shown). Thus, we postulated that the improved FHB resistance of A30 may be due to a chance background introgression of a major FHB resistance QTL from Freedom located elsewhere in 
the genome. A population of $90 \mathrm{~F}_{2: 3}$ families derived from the cross between Apogee and A30 was developed for genetic analysis and molecular mapping of FHB resistance identified in A30.

Plant growth conditions: To quantify the FHB resistance of A30 and to examine gene action, Apogee and A30 were crossed to produce $F_{1}$ seeds. In fall 2009, spring 2010, and fall 2010, both parents and $F_{1}$ plants were grown for FHB resistance evaluations. Seeds of each genotype were planted 6 to a "Magnum Square" pot $(15 \mathrm{~cm}(\mathrm{~L})$ x $15 \mathrm{~cm}(\mathrm{~W}) \times 16 \mathrm{~cm}(\mathrm{H})$ : Belden Plastics, St. Paul, MN) filled with a soilless growing media (Metro-Mix 200; Sun Gro, Agawam, MA), with 5 replicate pots per genotype, in a completely randomized design. Each pot was fertilized with 14N-14P-14K Osmocote (Scotts, Marysville, OH), and plants were grown in a greenhouse set to $20^{\circ} \mathrm{C}$ under a 16 hour (h) daylength.

To compare growth chamber results with those from the greenhouse, and to evaluate deoxynivalenol (DON) accumulation in Apogee and A30, seed of Apogee and A30 was sown, 6 to a pot, in Magnum Square pots containing Metro-Mix 200. Four pots of each genotype were sown for each of the three replicates of the experiment, with individual pots serving as the experimental unit. Pots were placed in an E8VH growth cabinet (Conviron, Winnipeg, Canada) set to $22^{\circ} \mathrm{C}$ and a $16 \mathrm{~h}$ daylength.

For molecular mapping of FHB resistance in A30, $90 \mathrm{~F}_{2: 3}$ families from the cross Apogee $\mathrm{x}$ A30 were planted for inoculations in fall 2009 and spring 2010 in the greenhouse. In both experiments, 18 seeds per $F_{2: 3}$ family were planted, 6 seeds to a pot in three Magnum Square pots containing Metro-Mix 200 potting mix. The three pots for each $\mathrm{F}_{2: 3}$ family were kept adjacent to each other during growth and considered a single experimental unit. Plants were grown under a $16 \mathrm{~h}$ daylength at $20^{\circ} \mathrm{C}$. To accommodate dew chamber space limitations, the $\mathrm{F}_{2: 3}$ population 
was divided into three equal sized groups of $\mathrm{F}_{2: 3}$ families with planting dates staggered over three dates separated by 4 to 5 days. Three pots of each parent were planted in each staggered planting date, and the greenhouse layout of lines was based on complete randomization within each planting date.

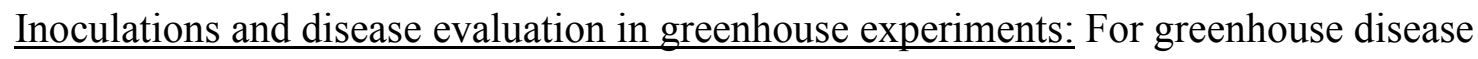
evaluations, flowering of all primary spikes of $\mathrm{F}_{2: 3}$ plants was tracked, and inoculations were completed at anthesis and up to 2 days later. Spikes were inoculated at a single floret located near the center of the spike. Inoculum consisted of $10 \mu \mathrm{L}$ of an aqueous suspension of macroconidia $\left(5 \times 10^{3}\right.$ spores total per inoculation, with $0.1 \%$ Triton X-100) of $F$. graminearum isolate Butte86ADA-11 (Evans et al. 2000). Inoculated plants were transferred to a dew chamber for $72 \mathrm{~h}$, and then returned to the greenhouse to allow disease symptoms to develop. Disease severity (\% of symptomatic spikelets) was recorded for each inoculated spike when the Apogee checks within each planting date reached a disease severity of approximately $85 \%$, and mean disease severity for each $\mathrm{F}_{2: 3}$ family and the parents was calculated using these data.

$\underline{\text { Inoculations and disease-related evaluations in growth chamber experiments: Disease }}$ evaluations in the growth chamber experiment consisted of inoculation of primary spikes at anthesis. Three of the four Apogee and A30 pots in each biological replicate were pointinoculated both in a central floret and in two other florets, one located two spikelets above and one two spikelets below the inoculated middle floret. In the fourth pot of each replicate, primary spikes of the Apogee and A30 plants were point-inoculated only in the central floret. Spikes were inoculated using the same protocol as described for greenhouse experiments, except that 
following inoculation the spikes were covered with clear polyethylene zip-seal bags for three days to simulate a prolonged dew period. Spikes from individual replicated pots of A30 and Apogee in which multiple florets were inoculated were harvested at 6,9 or 12 days after inoculation, and all the inoculated spikes from each individual replicate pot were pooled and lyophilized. The lyophilized tissue was milled to a powder and approximately $0.5 \mathrm{~g}$ of each was used for DON analysis by a modified method of Mirocha et al. (1998) as described in Fuentes et al. (2005). For the pots where spikes were inoculated in a single floret only, plants were allowed to mature, and inoculated spikes were then harvested and seeds were manually removed and the grain visually assessed and classified as either tombstone, shrunken, or sound.

Molecular marker analysis: A large scale screen of 1440 wheat genomic and EST-derived SSR markers from multiple sources was completed to identify polymorphisms between A30 and A31 for subsequent analysis within the mapping population. A31 is a FHB-susceptible $\mathrm{BC}_{4}$ sister line of A30 selected to harbor the Apogee allele of Xgwm296. The PCR conditions used for amplifying SSR primers followed the methods described by Tsilo et al. (2010). Fluorescent dyelabeled PCR fragments were separated on an ABI3130xl Genetic Analyzer (Applied Biosystems, Grand Island, NY). Genotype calling was performed using the software GeneMapper v3.7 (Applied Biosystems) followed by manual editing to confirm the accuracy of allele calls.

For molecular mapping of FHB resistance, DNA from Apogee, A30 and the $\mathrm{F}_{2}$ individuals from the Apogee x A30 mapping population was extracted by a version of the method described by Riede and Anderson (1996) designed for smaller scale extractions. Molecular markers polymorphic between A30 and A31 were re-evaluated for polymorphism between Apogee and A30; the markers typically were amplified by PCR using conditions detailed in the original 
publications reporting them. The PCR amplification products were resolved by vertical acrylamide gel electrophoresis, followed by silver staining using the methods described by Liu and Anderson (2003). Polymorphic markers were then scored for segregation in the mapping population.

Aneuploid analysis to locate molecular markers associated with FHB resistance was completed. DNA of Apogee, A30, and A31, together with DNA of Chinese Spring wheat (CS), CS chromosome 3D ditelosomic stocks, and CS chromosome arm 3DL segmental deletion lines 3DL-1 and 3DL-3 (Endo and Gill 1995), was used for PCR amplification of selected SSR markers to relate their physical locations on chromosome 3D, based on deletion bin mapping (Sourdille et al. 2004), to their genetic positions on a molecular map of the chromosome (Yu et al. 2009).

\section{Results}

Disease development in A30, Apogee and $\mathrm{F}_{1}$ hybrids: Following point inoculations, disease symptoms developed rapidly in Apogee as expected from prior research (Mackintosh et al. 2006), with disease severity reaching approximately 50\% within 10 days of inoculation, and nearly $80 \%$ by day 14 after inoculation. In contrast, A30 disease severity was less than half that of Apogee in these same time points. By day 18, disease severity in Apogee was approaching a plateau near $90 \%$, while A30 was approximately 50\%. By day 22, disease severity in A30 had reached 65\% while Apogee was 95\% (Fig. 1). The overall pattern of disease progression differed between the two genotypes as well. Apogee exhibited a curvilinear pattern often seen in FHB evaluations involving highly susceptible genotypes, while in A30 it was linear. The $\mathrm{F}_{1}$ 
hybrids exhibited disease severities that fell between the means of the two genotypes (Fig. 1), suggesting genetic additivity of the FHB resistance in A30.

Disease severity in Apogee x A30 F 2:3 families: In the fall 2009 greenhouse disease evaluation, inoculations within each of the three individual staggered $\mathrm{F}_{2: 3}$ family plantings were completed within three day windows, while in the spring 2010 experiment the comparable inoculations were completed in windows ranging between three and five days. This highlights the uniform flowering within the experimental population, as expected from a cross between a particular genotype and its $\mathrm{BC}_{4}$-derived near-isoline. Mean disease severities for $\mathrm{F}_{2: 3}$ families were obtained from a mean of 17.6 spikes (range 16-18) in the experiment completed in fall 2009, while in the spring 2010 experiment $F_{2: 3}$ family means were obtained from between 11 and 18 spikes (mean 17.4).

Apogee reached a disease severity of approximately 85\% 14 days after inoculation (dai) in the fall 2009 experiment, while it reached this level 15 dai in the spring 2010 experiment. Thus 14 dai and 15 dai were chosen as the rating times for inoculated spikes in fall 2009 and spring 2010, respectively. In the fall 2009 inoculation, the mean disease severity of Apogee (calculated from the disease severities of Apogee included in each of the three staggered plantings), was $86 \% 14$ dai, while A30 exhibited a disease severity of $35.2 \%$. The mean disease severity of the $\mathrm{F}_{2: 3}$ families in the fall 2009 experiment ranged from $28.1 \%$ to $97 \%$. In spring 2010 , parental disease severity means were $87.5 \%$ for Apogee and $41.8 \%$ for A30, while the range of mean severity in the $\mathrm{F}_{2: 3}$ families was between $30.6 \%$ and $100 \%$. The distribution of mean $\mathrm{F}_{2: 3}$ family disease severities in the two evaluations is shown in Fig $2 \mathrm{~A}$. A set of $16 \mathrm{~F}_{2: 3}$ families exhibited significantly higher levels of resistance than Apogee, and the proportion fit a monogenic ratio 
$\left(\mathrm{X}^{2}, \mathrm{P}>.10\right)$. The correlation between mean $\mathrm{F}_{2: 3}$ disease severities in the two evaluations was $0.844(\mathrm{P}<0.0001)$ (Fig. 2B).

Deoxynivalenol accumulation: A replicated growth chamber experiment was completed to obtain infected spike tissue for comparative analysis of DON accumulation in A30 and Apogee at three time points $(6,9,12$ dai) after point inoculation of three florets per spike. Results are shown in Fig. 3. Disease progression was notably more rapid in Apogee than A30 (Fig. 3A). A large difference in DON accumulation was evident between the two genotypes by the 6 dai time point, with Apogee spikes having accumulated approximately $80 \%$ more DON than A30. By the last sampled time point (12 dai), DON levels in Apogee spikes had increased $41 \%$ compared to the 6 dai samples, whereas the DON levels in A30 had increased just 23\% (Fig 3B). The level of DON detected in both Apogee and A30 ranged from $207 \mathrm{ug} \mathrm{g}^{-1}$ to $523 \mathrm{ug} \mathrm{g}^{-1}$, depending on the timepoint sampled and genotype (Table 1). While the range of concentrations is high compared to field studies, this is likely due to several factors including the extreme susceptibility of Apogee to FHB (Macintosh et al. 2006), the fact that the spikes were point inoculated at multiple locations, and the fact that DON concentrations in the entire spike, including the rachis which has been reported to have the highest DON levels in the spike (Cowger and Arellano 2013) and not just grain samples, were evaluated.

Within this same experiment, replicated single floret point-inoculated pots of Apogee and A30 were included to monitor disease development and progression, and for harvesting to evaluate grain quality. The disease severities at 9, 12 and 15 dai are shown in Table 1; the difference in resistance between Apogee and A30 was evident, except that disease severities were higher in this growth chamber study than in the greenhouse evaluations. At maturity, all 
seeds were removed from these single point-inoculated spikes and grouped into different classes - tombstone, shrunken, and sound. The relative distribution of these is shown in Table 1. Seeds harvested from A30 had a significantly lower proportion of tombstones (30\%) than did Apogee (71\%), as well as a higher percentage of sound grain (40.7\%, versus 5.5\% for Apogee).

Molecular mapping of FHB resistance in A30: A screen of 1440 wheat SSR markers identified 42 that were polymorphic between A30 and its susceptible sib line and Apogee surrogate, A31. Subsequent evaluation of these markers on silver-stained vertical acrylamide gels identified 24 of the polymorphisms that could be resolved between A30 and A31, and of these 15 were found to be polymorphic between A30 and Apogee. As expected there was no association between $\mathrm{Xgwm} 296$, the SSR marker reported to be linked to a major FHB resistance QTL in Freedom and thus selectively introgressed into A30, and FHB resistance segregation (data not shown), which supported our hypothesis that the resistance of A30 was due to a background introgression during its development.

Surprisingly, seven of the 15 markers that were polymorphic between Apogee and A30 exhibited a null phenotype in A30 despite the fact that both Apogee and Freedom displayed amplicons (Fig. 4), as did the FHB-susceptible sister line of A30, A31. Five of these markers (Xcfd223, Xwmc552, Xwm631, Xgwm3, and Xcnl093) were used to genotype the Apogee x A30 mapping population markers, which revealed that a set of $16 \mathrm{~F}_{2}$ individuals were the only plants to exhibit null phenotypes for these markers. An examination of the relationship between variation for $\mathrm{FHB}$ resistance in the $\mathrm{F}_{2: 3}$ families evaluated in the greenhouse and segregation for the collective null phenotype across markers revealed a strong association between the null marker phenotypes and greater levels of FHB resistance (Fig. 5). 
Since four of the markers exhibiting the null phenotype previously have been reported to map to chromosome $3 \mathrm{D}$, the results suggested the existence of a spontaneous deletion on this chromosome during the development of A30. To further substantiate this hypothesis, the markers for which both Apogee and Freedom had amplicons, but which were null in A30, were examined in Chinese Spring wheat, its chromosome 3D ditelosomic stocks, and two partial chromosome arm 3DL deletion lines (3DL-1 and 3DL-3). Results indicate that all of the markers reside in the terminal deletion (bin 3) of chromosome arm 3DL (Fig. 6), further supporting the presence of a spontaneous deletion from chromosome arm 3DL that arose during development of A30. An additional marker, $X b \operatorname{arc} 71$, which was reported as the most distal marker on chromosome arm 3DL in a previous study (Somers et al. 2004), was also evaluated in the Chinese Spring deletion stocks and was found to reside in chromosome arm 3DL bin 3. A30 exhibited a null phenotype for this marker as well, and when mapped in the Apogee $\mathrm{x} A 30 \mathrm{~F}_{2}$ mapping population, the Xbarc71 null phenotype co-segregated with the other markers exhibiting null phenotypes (Fig. 6).

To refine the size of the deletion breakpoint, additional markers developed for chromosome arm 3DL to genetically map a Hessian fly resistance gene (Yu et al. 2009) were employed to test for their presence or absence in Apogee, A30, and the Chinese Spring chromosome 3D deletion stocks. The results indicate that the chromosome breakpoint in A30 is between markers Xrwgs2 and Xrwgs 1 developed by $\mathrm{Yu}$ and colleagues for their study, which is in proximity to the deletion that defines bin 3 of this chromosome arm in Chinese Spring (Fig. 6). The deletion therefore spans approximately $35 \mathrm{cM}$ of chromosome arm 3DL, and represents approximately $19 \%$ of the length of this chromosome arm. 


\section{Discussion}

Intensive research efforts to identify genes or QTLs for FHB resistance in wheat have identified a large number of QTLs, but most exhibit only small to modest effects (Buerstmayr et al. 2009). Only one of the dozens of reported FHB resistance QTLs, Fhb1, is broadly employed by most breeding programs to complement phenotypic selection in the field. Thus, any new QTLs with potentially large and repeatable effects would be highly valuable for improving FHB resistance in wheat. In this study, we identified a $\mathrm{BC}_{4}$-derived NIL of the highly susceptible wheat cultivar Apogee that exhibited a significant improvement in Type II FHB resistance. We speculated that the resistance in this NIL, A30, was due to the chance background introgression of a new major FHB resistance QTL from the soft red winter wheat Freedom. However, molecular mapping revealed that the resistance stems from the loss of the terminal region of chromosome arm 3DL. The deletion occurred during the development of A30, since neither Apogee nor Freedom possess it.

Our results suggest that the deletion present in A30 eliminates a gene conferring susceptibility to FHB. Currently, published studies of the genetics of FHB susceptibility are sparse. Type II FHB resistance was evaluated in ditelosomic stocks of Chinese Spring wheat, which is considered moderately resistant (Ma et al. 2006). Several of the lines exhibited greater susceptibility than Chinese Spring, which may be due to the deletion of resistance QTLs or genes essential to the host defense response against $F$. graminearum. Interestingly, nearly the same number of the lines exhibited higher levels of resistance than Chinese Spring, with some exhibiting as much as a 75\% reduction in FHB severity, which implies that genes for FHB susceptibility are present on a number of wheat chromosomes. In tetraploid wheat, chromosome 
2A, from the wild emmer line Israel A, was found to increase FHB susceptibility significantly when substituted into the moderately susceptible durum wheat cultivar Langdon (Stack et al. 2002). This observation was attributed to the presence of FHB susceptibility genes or possible resistance inhibitor genes on this chromosome, since Israel A itself is highly susceptible to FHB even though it is the source of the FHB resistance QTL Qfhs.ndsu-3A (Otto et al. 2002). Subsequent molecular mapping confirmed that a region of Israel A chromosome 2A was associated with increased FHB susceptibility (Garvin et al. 2009).

Results of some genetic analyses also may suggest the possible existence of FHB susceptibility genes, although alternative explanations cannot be ruled out. Mapping FHB resistance in wheat populations developed from crosses between Sumai 3 (a source of Fhbl) and different highly susceptible parents revealed that the susceptible allele for a QTL on chromosome arm 2DS came from Sumai 3 and not the susceptible parents (Basnet et al. 2012). However, this QTL is located in close proximity to the dwarfing gene Rht8 (Handa et al. 2008) which is known to be associated with FHB susceptibility (Mao et al. 2010). Background genetic effects on the effect of Fhbl were reported by Pumphrey et al. (2007), who found that fewer than half of a series of hard red spring wheat isoline pairs differing for the presence of Fhbl exhibited significant differences in Type II resistance. Similarly, Salameh et al. (2011) reported that the relative effect of $F h b 1$ and the Type I FHB resistance QTL $Q f h s . i f a-5 A$ varied considerably, depending on the winter wheat genetic background in which they were present. These findings may reflect the presence of susceptibility genes, or alternatively, the presence of genes that inhibit resistance QTLs or the absence of accessory genes needed for their complete expression.

In our study, the reduction in FHB severity in A30 compared to Apogee ranged between $31 \%$ and $59 \%$ depending on the time point at which FHB severity was evaluated. The analysis 
of FHB resistance in Chinese Spring deletion lines (Ma et al. 2006) did not include the ditelosomic line lacking chromosome arm 3DL, so no comparison can be made to that study to determine if the loss of this region of the wheat genome enhances resistance in different genetic backgrounds. However, in that study the loss of chromosome arm 3BL from Chinese Spring dramatically increased resistance, while the loss of chromosome arm 3AL decreased resistance. Thus, chromosome arms 3BL and 3DL may harbor homoeoalleles for FHB susceptibility. Based on the fact that no Type II FHB resistance QTL has been mapped to the particular region of chromosome arm 3DL that is missing in A30 (Buerstmayr et al. 2009), it does not appear as though there is naturally-occurring genetic variation for the gene or genes in this region affecting FHB resistance responses in wheat.

Our research revealed that not only is Type II FHB resistance enhanced upon deletion of the terminal region of chromosome arm 3DL, but DON accumulation is also reduced. That this occurs in a highly susceptible background is reminiscent of research in other pathosystems involving necrotrophic fungi. For instance, susceptibility to the toxin SnTox1 produced by Stagonospora nodorum can be eliminated by loss of wheat chromosome 1B (Liu et al. 2004). Similarly, insensitivity to Pyrenophora triticii-repens ToxA is associated with the deletion of the gene $T s n 1$ (Faris et al. 2010). For many other pathosystems involving necrotrophic pathogens, resistance is often governed by recessive genes, in contrast to most resistance genes for biotrophic pathogens that are typically classical R-genes (Mengiste 2012). The difference is attributed to the fact that necrotrophs must kill host cells to obtain nutrients, and so produce toxins to accomplish this. Host resistance is gained when host toxin targets are structurally modified and no longer allow the toxins to initiate cell death. DON is the best-characterized virulence factor of $F$. graminearum, although others exist (eg. Voigt et al. 2005; Menke et al. 
2012). DON targets ribosomal protein L3, leading to perturbation of protein synthesis and ultimately cell death, and other targets of DON may exist (Feinberg and McLaughlin 1989). It is possible that the increased FHB resistance observed in A30 may be associated with the elimination of a gene encoding a target of DON or another virulence factor produced by $F$. graminearum. This would result in reduced host cell death and be accompanied by a commensurate reduction in the rate of colonization of the spike by the fungus. Ethylene signaling in wheat spikes during infection by $F$. graminearum appears to play a role in FHB susceptibility, and interfering with ethylene signaling increases resistance (Chen et al. 2009). Gardiner et al. (2010) proposed that polyamine biosynthesis by the host plant is associated with the induction of DON by F. graminearum. Thus it is possible that the deletion of the 3DL segment removes a gene associated with one of these metabolic processes, leading either to reduced ethylene-induced cell death, or reduced synthesis of polyamines that reduce the induction of DON, either of which ostensibly could interfere with $F$. graminearum virulence, leading to impeded colonization of the spike. Our results suggest that, as opposed to other welldefined pathosystems involving necrotrophs, the gene or genes deleted in A30 that result in enhanced FHB resistance act in a dose-dependent manner. A more refined understanding of $F$. graminearum and its interaction with and manipulation of host metabolic processes will shed more light on other potential host metabolic processes that might be targets of modification to protect against $F$. graminearum virulence factors.

Disease resistance is complex and can involve many different passive and active defenses. Development of FHB-resistant wheat has proceeded more slowly than might be expected, because despite global efforts there is a dearth of FHB resistance genes or major FHB resistance QTLs that can be pyramided via marker-assisted selection in wheat germplasm to obtain high 
levels of resistance. There has been much discussion of developing novel strategies for controlling FHB, including removal of susceptibility factors or modification of potential targets of $F$. graminearum virulence factors. But to date little has been done in this arena of FHB research, in part because of the challenge of differentiating between the presence of a susceptibility gene and the absence of a resistance QTL. Our study demonstrates the presence of a gene or genes on the long arm of chromosome 3DL that, when removed, results in a dramatic improvement of FHB resistance. We postulate that this gene is likely to condition some degree of susceptibility to FHB in a manner that may be similar to genes conferring sensitivity to other necrotrophic fungi. Transfer of this deletion to other genotypes will determine if the resistance enhancement effect is unique to Apogee or occurs in other genetic backgrounds. We note that a preliminary analysis of Chinese Spring chromosome arm 3DL deletion lines suggests that elimination of part of this arm also appears to increase FHB resistance (data not shown), and so we speculate that the deletion in A30 will also increase FHB resistance in other genotypes. Further, though the deletion is large, it is worth examining its agronomic effect; if there is a minimal detrimental impact, the deletion may be a novel resource to increase FHB resistance. While there is still only a small amount of evidence supporting the existence of genes that confer FHB susceptibility, additional research employing mutagenesis of susceptible genotypes may provide an avenue for identifying additional susceptibility genes and their locations, and deploying the mutated forms for genetic improvement of FHB resistance in a manner complementary to the use of resistance QTLs. Gain-of-resistance mutants from susceptible genotypes will also be a useful resource for exploring the biology of FHB susceptibility in wheat and its interaction with $F$. graminearum. 


\section{Acknowledgements}

The authors wish to thank Dr. Yanhong Dong for providing DON analysis for this study. This material is based upon work supported by the U.S. Department of Agriculture. This is a cooperative project with the U.S Wheat and Barley Scab Initiative. 


\section{References}

Bai, G., and Shaner, G. 2004. Management and resistance in wheat and barley to Fusarium head blight. Ann. Rev. Phytopathol. 42: 135-161.

Basnet, B., Glover, K.D., Ibrahim, A.M.H., Yen, Y., and Chao, S. 2012. A QTL on chromosome 2DS of 'Sumai 3' increases susceptibility to Fusarium head blight in wheat. Euphytica, 186: 91-101.

Buerstmayr, H., Ban, T., and Anderson, J.A. 2009. QTL mapping and marker-assisted selection for Fusarium head blight resistance in wheat: a review. Plant Breed. 128: 1-26.

Bugbee, B., Koerner, G., Albrechtsen, R., Dewey, W., and Clawson, S. 1997. Registration of 'USU-Apogee' wheat. Crop Sci. 37: 626.

Chen, X., Steed, A., Travella, S., Keller, B., and Nicholson, P. 2009. Fusarium graminearum exploits ethylene signaling to colonize dicotyledonous and monocotyledonous plants. New Phytol. 182: 975-983.

Cowger, C. and Arellano C. 2013. Fusarium graminearum infection and deoxynivalenol concentrations during development of wheat spikes. Phytopathol. 103: 460-471.

Cuthbert, P.A., Somers, D.J., and Brulé-Babel, A. 2007. Mapping of Fhb2 on chromosome 6BS: a gene controlling Fusarium head blight field resistance in bread wheat (Triticum aestivum L.). Theor. Appl. Genet. 114: 429-437.

Endo, T.R. and Gill, B.S. 1995. The deletion stocks of common wheat. J. Hered. 87: 295-307.

Evans, C.K., Xie, W., Dill-Macky, R., and Mirocha, C.J. 2000. Biosynthesis of deoxynivalenol in spikelets of barley inoculated with macroconidia of Fusarium graminearum. Plant Dis. 84: 654-660. 
Faris, J.D., Zhang, Z., Lu, H., Lu, S., Reddy, L., Cloutier, S., Fellers, J.P., Meinhardt, S.W., Rasmussen, J.B., Xu, S.S., Oliver, R.P., Simons, K.J., and Friesen, T.L. 2010. A unique wheat disease resistance-like gene governs effector-triggered susceptibility to necrotrophic pathogens. Proc. Natl. Acad. Sci. USA 107: 13544-13549.

Feinberg, B., and McLaughlin, C.C. 1989. Biochemical mechanism of action of tricothecene mycotoxins. In Tricothecene mycotoxicosis: pathophysiological effects. Vol. 1. Edited by V.R. Beasley. CRC Press, Boca Raton, FL. pp. 27-35.

Fuentes, R.G., Mickelson, H.R., Busch, R.H., Dill-Macky, R., Evans, C.K., Thompson, W.G., Wiersma, J.V., Xie, W., Dong, Y., and Anderson, J.A. 2005. Resource allocation and cultivar stability in breeding for Fusarium head blight resistance in spring wheat. Crop Sci. 5: 19651972.

Gardiner, D.M., Kazan, K., Praud, S., Torney, F.J., Rusu, A., and Manners, J.M. 2010. Early activation of wheat polyamine biosynthesis during Fusarium head blight implicates putrescine as an inducer of tricothecene mycotoxin production. BMC Plant Biol. 10: 289. doi:10.1186/1471-2229-10-289.

Garvin, D.F., Stack, R.W., and Hansen, J.M. 2009. Quantitative trait locus mapping of increased Fusarium head blight susceptibility associated with a wil emmer wheat chromosome. Phytopathol. 99: 447-452.Groth, J.V., Ozmon, E.A., and Busch, R.H. 1999. Repeatability and relationship of incidence and severity measures of scab of wheat caused by Fusarium graminearum in inoculated nurseries. Plant Dis. 83: 1033-1038.

Gupta, A. 2002. Host plant resistance genes to Fusarium head blight of wheat: sources, inheritance, and determination of genetic control. Ph.D. thesis. The Ohio State University. 
Handa, H., Namiki, N., Xu, D., and Ban, T. 2004. Dissecting of the FHB resistance QTL on the short arm of chromosome 2D using a comparative genomic approach: from QTL to candidate gene. Mol. Breed. 22: 71-84.

Liu, S. and Anderson, J.A. 2003. Marker-assisted evaluation of Fusarium head blight resistant wheat germplasm. Crop Sci. 43: 760-766.

Liu S., Hall, M.D., Griffet, C.A., and McHendry, A.L. 2009. Meta-analysis of QTL associated with Fusarium head blight resistance in wheat. Crop Sci. 49: 1955-1968.

Liu, Z.H., Faris, J.D., Meinhardt, S.W., Rasmussen, J.B., and Friesen, T.L. 2004. Genetic and physical mapping of a gene conditioning sensitivity in wheat to a partially purified hostselective toxin produced by Stagonospora nodorum. Phytopathol. 94:1056-1060.

Loffler, M., Schon, C., and Miedaner T. 2009. Revealing the genetic architecture of FHB resistance in hexaploid wheat (Triticum aestivum L.) by QTL meta-analysis. Mol. Breed. 23: $473-488$.

Ma, H., Bai, G., Gill, B.S., and Hartl, L.P. 2006. Deletion of a chromosome arm altered wheat resistance to Fusarium head blight and deoxynivalenol accumulation in Chinese Spring. Plant Dis. 90: 1545-1549.

McMullen, M., Bergstrom, G., DeWolf, E., Dill-Macky, R., Hershman, D., Shaner, G., amd Van Sanford, D. 2012. A unified effort to fight an enemy of wheat and barley: Fusarium head blight. Plant Dis. 96: 1712-1728.

McMullen, M.P., Jones, R., and Gallenberg, D. 1997. Scab of wheat and barley: a re-emerging disease of devastating impact. Plant Dis. 81: 1340-1348. 
Mackintosh, C.A., Garvin, D.F., Radmer, L.E., Heinen, S.J., and Muehlbauer, G.J. 2006. A model wheat cultivar for transformation to improve resistance to Fusarium head blight. Plant Cell Rep. 25: 313-319.

Mao, S., Wei, Y., Coao, W., Lan, X., Yu, M., Chao, Z., Chen, G., and Zheng, Y. 2010. Confirmation on the relationship between plant height and Fusarium head blight resistance in wheat (Triticum aetivum L.) by QTL meta-analysis. Euphytica 174: 343-356.

Mengiste, T. 2012. Plant immunity to necrotrophs. Annu. Rev. Phytopathol. 50: 267-294.

Menke, J., Dong, Y., and Kistler, H.C. 2012. Fusarium graminearum Tri12p influences virulence to wheat and tricothecene accumulation. Mol. Plant Microbe Inter. 25: 1408-1418.

Miedaner R., Reinbrecht, C., Lauber, U., Schollenberger, M., and Geiger, H.H. 2001. Effects of genotype and genotype $\mathrm{x}$ environment interaction on deoxynivalenol accumulation and resistance to Fusarium head blight in rye, triticale, and wheat. Plant Breed. 120: 97-105.

Mirocha, C.J., Kolaczkowski, E., Xie, W., Yu, H., and Jelen, H. 1998 Analysis of deoxynivalenol and its derivatives (batch and single kernel) using gas chromatography/mass spectrometry. J. Agric. Food Chem. 46:1414-1418.

Otto, C.D., Kianian, S.F., Elias, E.M., Stack, R.W., and Joppa, L.R. 2002. Genetic dissection of a major Fusarium head blight QTL in tetraploid wheat. Plant Mol. Biol. 48: 625-632.

Pumphrey, M.O., Bernardo, R., and Anderson, J.A. 2007. Validating the Fhbl QTL for Fusarium head blight resistance in near-isogenic wheat lines developed from breeding populations. Crop Sci. 47: 200-206.

Riede, C.R., and , J.A. 1996. Linkage of RFLP markers to an aluminum tolerance gene in wheat. Crop Sci. 36: 905-909. 
Salameh, A., Buerstmayr, M., Steiner, B., Neumayer, A., Lemmens, M., and Buerstmayr, H. 2011. Effects of introgression of two QTL for Fusarium head blight resistance from Asian spring wheat by marker-assisted backcrossing into European winter wheat on Fusarium head blight resistance, yield and quality traits. Mol. Breed. 28: 485-494.

Schroeder, H.W., and Christensen, J.J. 1963. Factors affecting resistance of wheat to scab caused by Gibberella zeae. Phytopathol. 53: 831-838.

Somers, D.J., Isaac, P., and Edwards, K. 2004. A high-density microsatellite consensus map for bread wheat (Triticum aestivum L.). Theor. Appl. Genet. 109: 1105-1114.

Sourdille, P., Singh, S., Cadalen, T., Brown-Guidera, G.L., Gay, G., Qi, L., Gill, B.S., Dufour, P., Murigneux, A., and Bernard, M. 2004. Microsatellite-based deletion bin system for the establishment of genetic-physical map relationships in wheat (Triticum aestivum L.). Funct. Integr. Genomics, 4: 12-25.

Srinivasachary, G.N., Steed, A., Simmonds, J., Leverington-Waite, M., Wang, Y., Snape, J., and Nicholson, P. 2008. Susceptibility to Fusarium head blight is associated with the Rht-D1b semidwarfing allele in wheat. Theor. Appl. Genet. 116: 1145-1153.

Stack, R.W., and McMullen, M.P. 1985. Head blighting potential of Fusarium species associated with spring wheat heads. Can. J. Plant Pathol. 7: 79-82.

Stack, R.W., Elias, E.M., Mitchell, F.J., Miller, J.D., and Joppa, L.R. 2002. Fusarium head blight reaction of Langdon durum-Triticum dicoccoides chromosome substitution lines. Crop Sci. 42: $637-642$.

Tsilo, T.J., Hareland, G.A., Simsek, S., Chao, S., and Anderson, J.A. 2010. Genome mapping of kernel characteristics in hard red spring wheat breeding lines. Theor. Appl. Genet. 121: 7171730. 
Voigt, C.A., Schafer, W., and Salomon, S. 2005. A secreted lipase of Fusarium graminearum is a virulence factor required for infection of cereals. Plant J. 42: 364-375.

Windels, C.E. 2000. Economic and social impacts of Fusarium head blight: changing farms and rural communities in Northern Great Plains. Phytopathol. 90: 17-21.

Xue, S., Li, G., Jia, H., Xu, F., Lin, F., Tang, M., Wang, Y., An, X., Xu, H., Zhang, L., Kong, Z., and Ma, Z. 2010. Fine mapping Fhb4, a major QTL conditioning resistance to Fusarium infection in bread wheat (Triticum aestivum L.). Theor. Appl. Genet. 121: 147-156.

Yu, G.T., Cai, X., Harris, M.O., Gu, Y.Q., Luo, M., and Xu, S.S. 2009. Saturation mapping of the genomic region harboring Hessian fly resistance gene $H 26$ in wheat. Theor. Appl. Genet. 118: 1589-1599. 
Table 1. Fusarium head blight (FHB) severity at 9, 12 and 15 days after inoculation (dai), and grain quality in single point-inoculated spikes of Apogee and A30, under growth chamber conditions.

\begin{tabular}{cccccccc} 
& \multicolumn{2}{c}{ FHB Severity $(\%)$} & & \multicolumn{3}{c}{ Grain Quality $(\%)^{a}$} \\
\cline { 2 - 3 } \cline { 6 - 8 } Genotype & 9 dai & 12 dai & 15 dai & & Sound & Shriveled & Tombstone $^{b}$ \\
\hline Apogee & $72.1^{\mathrm{b}}$ & 86.4 & 94.1 & & 5 & 23 & 71 \\
A30 & 39.6 & 53.3 & 75.9 & & 41 & 30 & 30
\end{tabular}

${ }^{a}$ Values are the means of the three biological replicates. Summed values for grain quality differ from $100 \%$ due to rounding error.

${ }^{b}$ Grain classified as shriveled has not filled normally, while tombstone grain is not only shriveled but also exhibits a chalky white, gray or pink appearance due to infection by Fusarium graminearum. 


\section{Figure Legends}

Figure 1. Temporal progression of Fusarium head blight (FHB) symptoms in point-inoculated Apogee, $\mathrm{A} 30$ and $\mathrm{F}_{1}$ hybrids. Values represent the mean of three experimental replicates. Bars represent standard errors.

Figure 2. A) Distribution of mean Fusarium head blight (FHB) severity values in Apogee x A30 $\mathrm{F}_{2: 3}$ families in two greenhouse disease evaluations. B) Relationship between mean FHB severity values in Apogee x A30 F $2: 3$ families in 2009 and 2010, with trend line shown.

Figure 3. A) Disease spread in representative spikes of Apogee and A30 inoculated in three florets per spike, under growth chamber conditions, 6, 9, and 12 days after inoculation (dai). Black dots on spikes indicate the inoculated florets on each spike B) Deoxynivalenol (DON) accumulation at 6,9 , and 12 days after point inoculation of the three florets. Values given are the mean of three replicates. Bars represent standard errors.

Figure 4. Examples of simple sequence repeat marker phenotypes for which null alleles are present in A30 but not in the parental lines Apogee or Freedom, nor the susceptible sister line A31. Ap, Apogee; Fr, Freedom.

Figure 5. Fusarium head blight (FHB) severity distribution in $\mathrm{F}_{2: 3}$ families from the cross $\mathrm{A} 30 \mathrm{x}$ Apogee, relative to the presence or absence of the chromosome arm 3DL deletion in A30, as determined by null marker phenotypes in the $F_{2}$ parents of the $F_{2: 3}$ families. Plotted values are 
the mean of the two independent greenhouse evaluations. Arrows indicate parental control mean FHB severities. Ap, Apogee.

Figure 6. Extent of chromosome deletion in A30, based on selected markers mapping to the long arm of chromosome 3D (Yu et al. 2009) (left side of figure), and physical locations of the markers based on marker locations in aneuploid stocks of Chinese Spring wheat chromosome arm 3DL. + indicates the presence of a marker; - indicates that the marker is absent. Ch Spr, Chinese Spring wheat; 3DL-3, Chinese Spring deletion line 3DL-3; 3DL-1, Chinese Spring deletion line 3DL-1. Chromosome breakpoints for the deletion lines are shown on the chromosome arm 3DL graphic, together with inferred chromosome arm 3DL bin locations of markers on the map. 


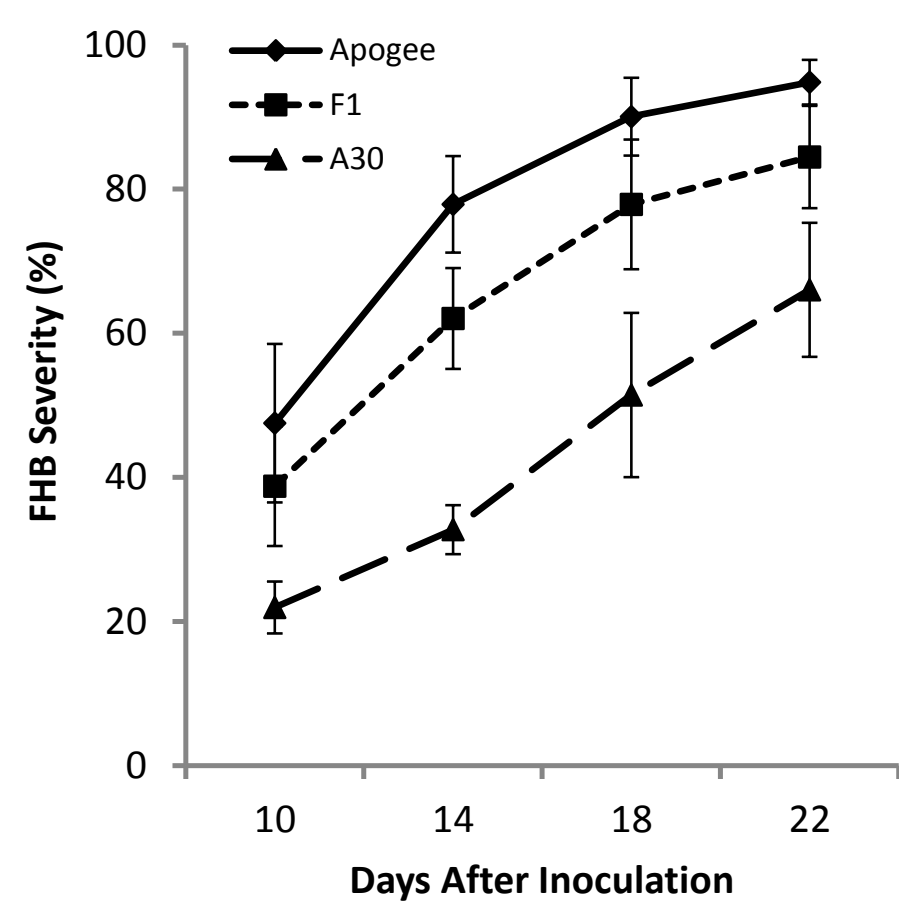



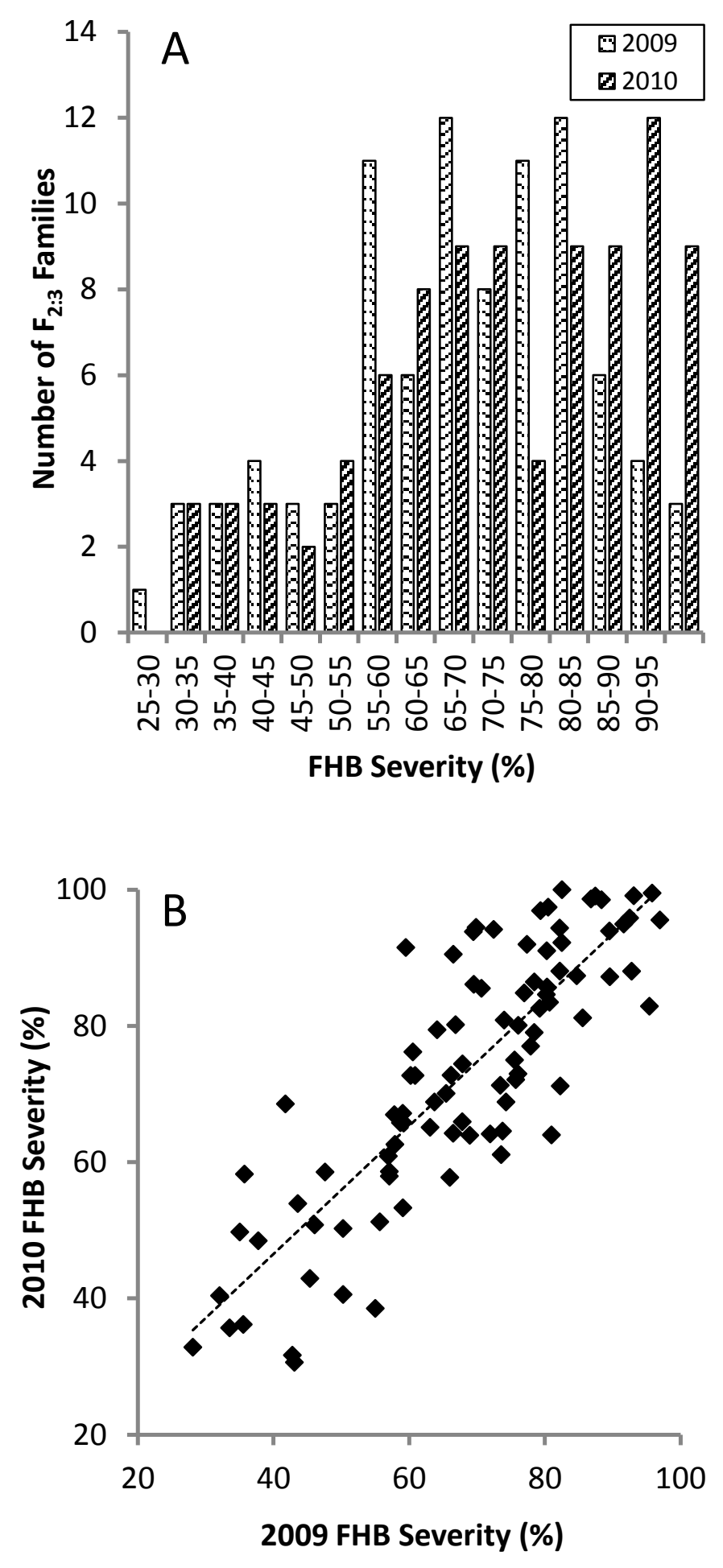

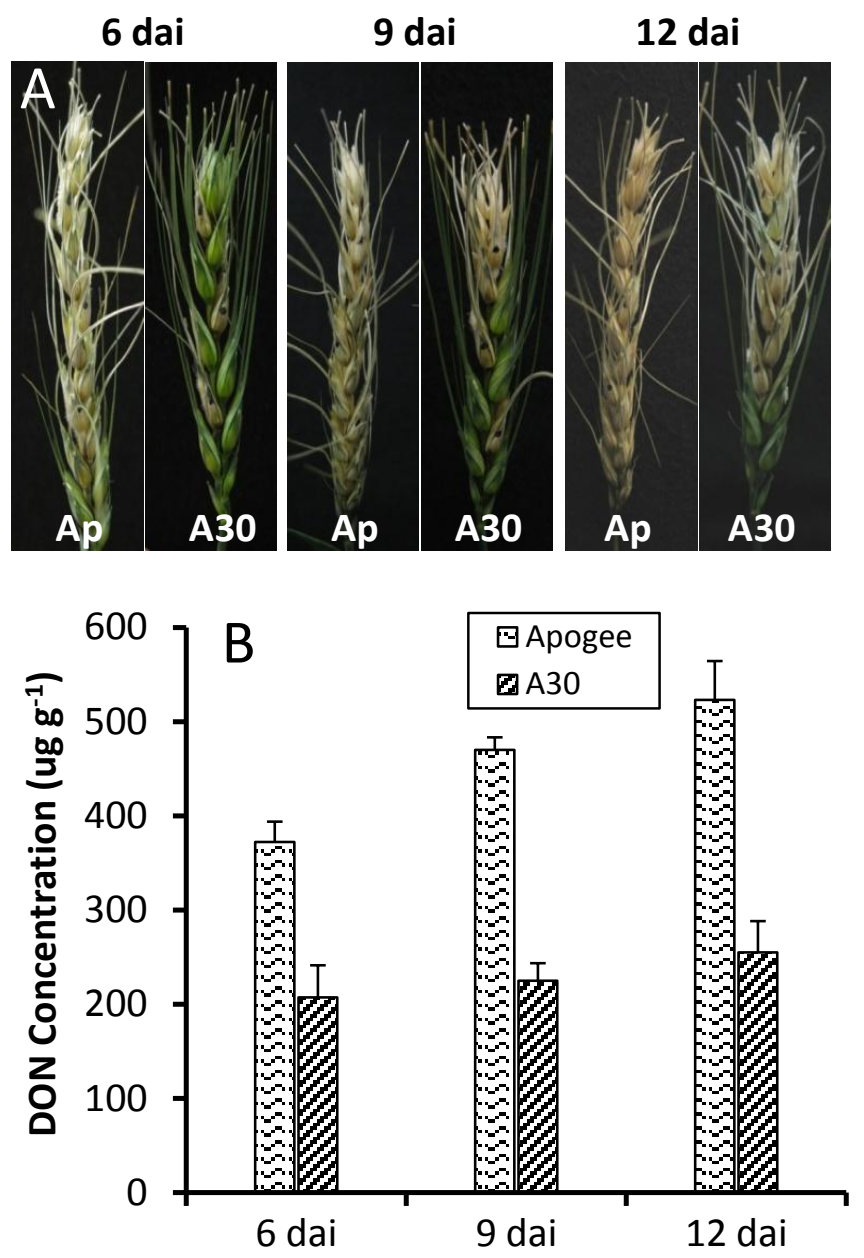


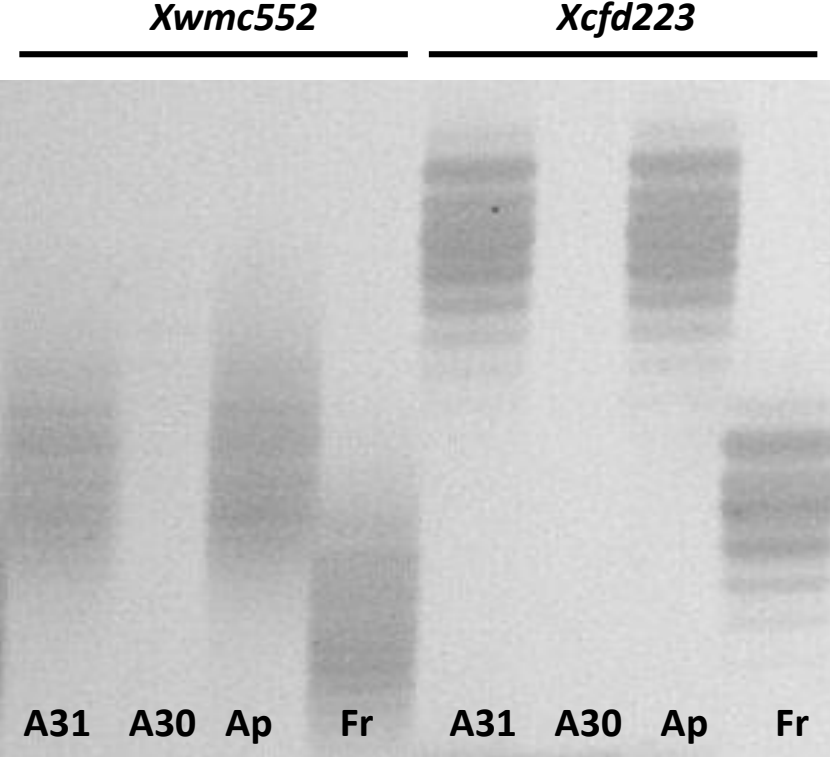




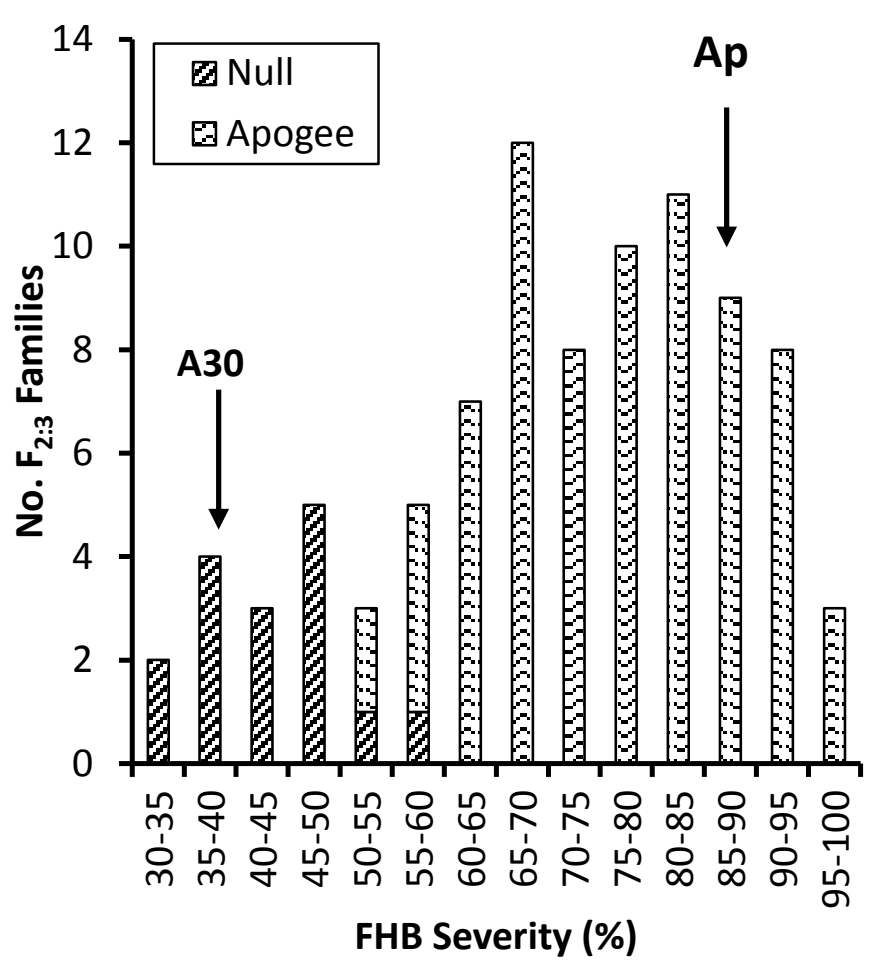




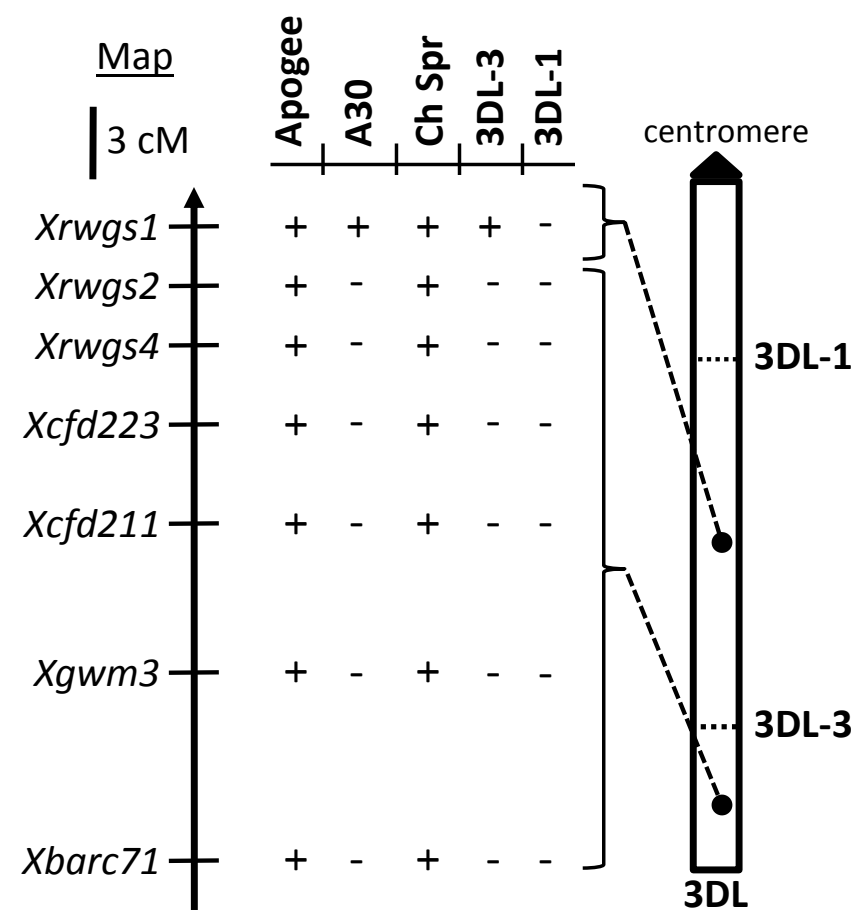

